This item was submitted to Loughborough's Research Repository by the author.

Items in Figshare are protected by copyright, with all rights reserved, unless otherwise indicated.

\title{
MetaLib and SFX at Loughborough University Library.
}

PLEASE CITE THE PUBLISHED VERSION

PUBLISHER

(C) Emerald

LICENCE

CC BY-NC-ND 4.0

REPOSITORY RECORD

Stubbings, Ruth. 2019. "Metalib and SFX at Loughborough University Library.". figshare. https://hdl.handle.net/2134/175. 


\section{MetaLib and SFX at Loughborough University Library}

Ruth Stubbings

\section{The author}

Ruth Stubbings is Academic Services Manager, Pilkington Library, Loughborough University, UK.

E-mail: r.e.stubbings@lboro.ac.uk

\section{Keywords}

Libraries, Universities, Library management, Information systems

\section{Abstract}

This article outlines the implementation of MetaLib and SFX at Loughborough University. It includes details of the timescale, work and staff involved, how the services work, usage and the reactions of readers.

\section{Electronic access}

The Emerald Research Register for this journal is available at http://www.emeraldinsight.com/researchregister

The current issue and full text archive of this journal is available at

http://www.emeraldinsight.com/0305-5728.htm
VINE: The Journal of Information and Knowledge Management Systems Volume $33 \cdot$ Number $1 \cdot 2003 \cdot$ pp. 25-32

(C) MCB UP Limited · ISSN 0305-5728

DOI $10.1108 / 03055720310488293$

\section{Introduction}

In January 2001 Loughborough University Library purchased a new library management system, Aleph from Ex Libris[1]. At the same time the Library purchased Aleph's sister products, SFX and MetaLib.

\section{What are MetaLib and SFX?}

MetaLib and SFX are two separate products that complement each other by managing access to databases and journals. MetaLib[2] is a tool that enables a library to provide a single access point (portal/gateway) to Web databases, with simultaneous multiple database searching functionality for those databases that allow Z39.50 searching. Thus the Library can provide subject lists of the 170 databases to which it subscribes via MetaLib and readers can often search more than one database at a time. (For a more detailed summary of the different types of commercial portals available see Cox and Yeates (2002).)

SFX[3] complements MetaLib by linking directly to the full text of e-journals or to the catalogue record of print journals to which the Library subscribes, either from MetaLib or other databases that utilise OpenURL functionality. To get started a library has to request that OpenURL-aware publishers activate SFX on their databases. When activated a text link or SFX icon appears next to each bibliographic record on the database.

Readers can click on this link and data from the bibliographic record are sent to the SFX server to check whether the Library subscribes to that item. If the Library does have a subscription to the item, then an SFX menu will provide hotlinks to the full text and/or library catalogue record. To date the Library subscribes to over 3,500 e-journals and approximately 2,000 print journals.

The author would like to thank all Library staff who were involved in the implementation of MetaLib and SFX, and in particular Jeff Brown, Frank Parry, Gary Brewerton and Dawn Cole for reading and checking that this article was factually and grammatically correct. Also thanks to Ex Libris for giving permission for the screenshots of MetaLib to be included in the article. 


\section{Implementation of MetaLib}

Unlike the University of East Anglia (Lewis, 2002), the Pilkington Library took a large team approach to the implementation of MetaLib. The MetaLib Group's membership included representatives from the Systems Team, Support Services Team (who arrange subscriptions to the databases) and all nine academic librarians. The academic librarians are the main liaison point with the academic departments, staff the enquiry desks and deliver information literacy training. It was therefore felt that they should play a pivotal role in the development of MetaLib, giving them a sense of ownership. The negative side to this arrangement was that decision making by committee could be slow.

The group spent a lot of time discussing:

- The look and feel of MetaLib. It was agreed to make minor changes to terminology, e.g. e-shelf was changed to basket, but the product still generally looks as it came out of the box.

- Issues of branding. Several academic librarians proposed changing the name of MetaLib to make it more identifiable as a Loughborough University service; for example, Curtin University in Australia has renamed MetaLib as Gecko[4]. The Library held a competition to rename MetaLib, but none of the entries was considered to be an improvement. To identify MetaLib as a service being offered by the Library, the Library's logo was added to the top banner of MetaLib.

- Contents of MetaLib. It was agreed that all databases should be included on MetaLib, no matter in what format, e.g. Web-based, networked CD-ROMs available on the campus network and stand alone CDROMs only available in the Library. This was because there were strong concerns that, if they were not included, they would be overlooked by students. It was also agreed that pertinent print abstracts would also be placed on MetaLib, e.g. Chemical Abstracts.

- How the databases should be listed on MetaLib. MetaLib allows databases to be grouped together in sections known as information gateways (see Figure 1). It was agreed that the information gateways would reflect the subject guides already in existence on the Library's Web site. There was one major problem with this approach. The current version of MetaLib only allows a database to belong to five information gateways and the Library has several databases that could belong to more than five information gateways. Thankfully the Systems Team developed a way round this by creating a series of dummy information gateways and linking these to multiple true gateways. For example, a dummy gateway of Engineering was created that linked to all the Engineering Department gateways.

- Future of some of the Library's Web pages. It was agreed to discontinue the subject guides as there was no point in maintaining two indexes. But the $\mathrm{A}-\mathrm{Z}$ list of databases would be maintained for at least a year until the reliability of MetaLib was confirmed. This would also allow the departments time to gain familiarity with using MetaLib as the major route into the databases.

- Information literacy skills. Some academic librarians had reservations, feeling that MetaLib may encourage poor searching techniques by students. The Group agreed that during training sessions MetaLib would be put forward as the quick and easy way to find information for assignments, but that, for detailed projects or dissertations, an individual database's own interfaces should be searched, thus allowing complex search strategies to be performed.

\section{Cataloguing and configuring resources on MetaLib}

As indicated above, MetaLib behaves as a portal to resources and provides one searching interface for databases that are Z39.50-

compliant and therefore "cross-searchable". All resources have to be catalogued on MetaLib. This is an easy task as Ex Libris provide cataloguing forms (see Figure 2). They also have a knowledge base of many of the major resources and therefore these items do not have to be catalogued from scratch - they just need to be activated and tidied up.

For databases to be "cross-searchable" they have to be configured. Again Ex Libris have already configured some of the major databases 


\section{Figure 1 Search screen}

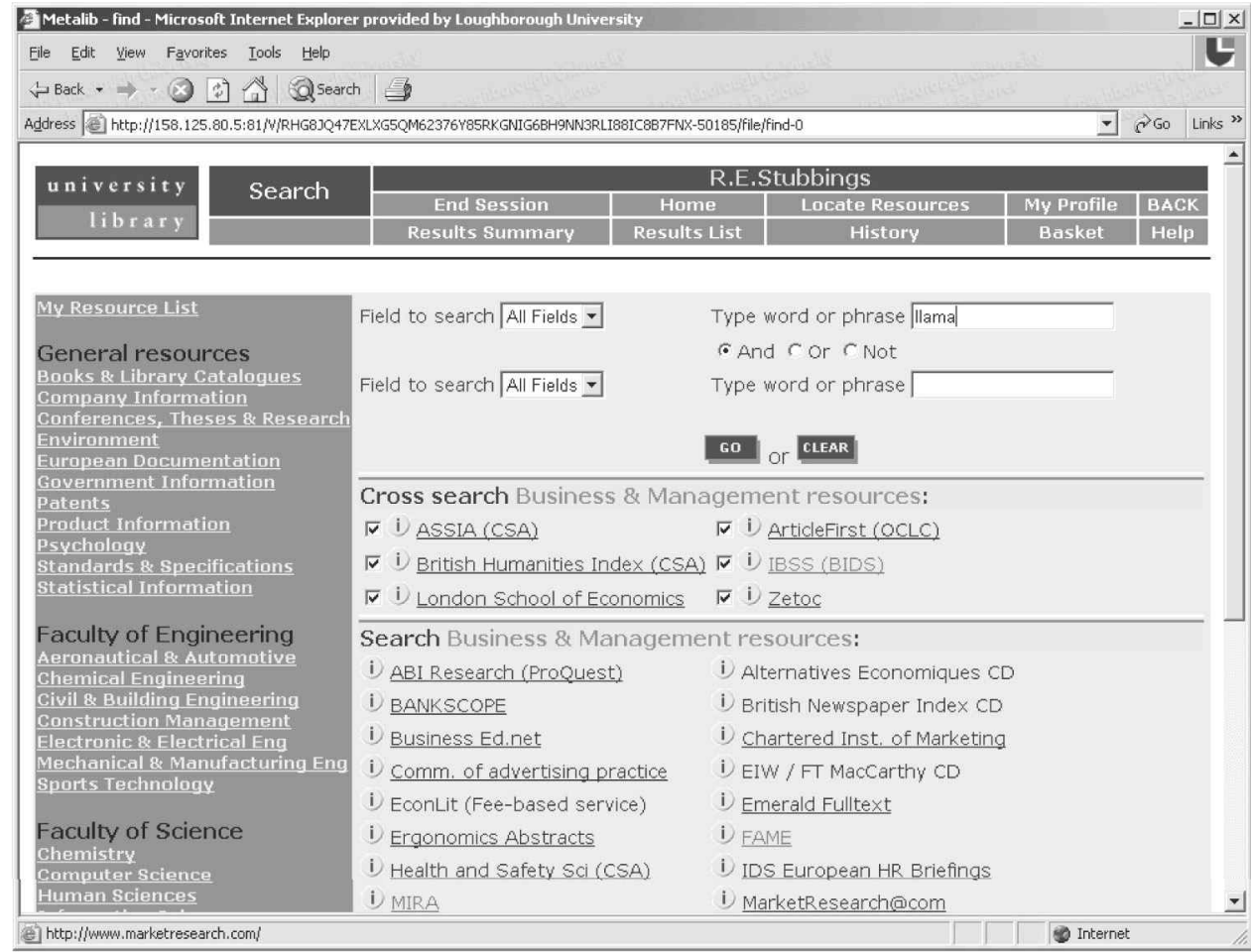

\section{Figure 2 Cataloguing record}

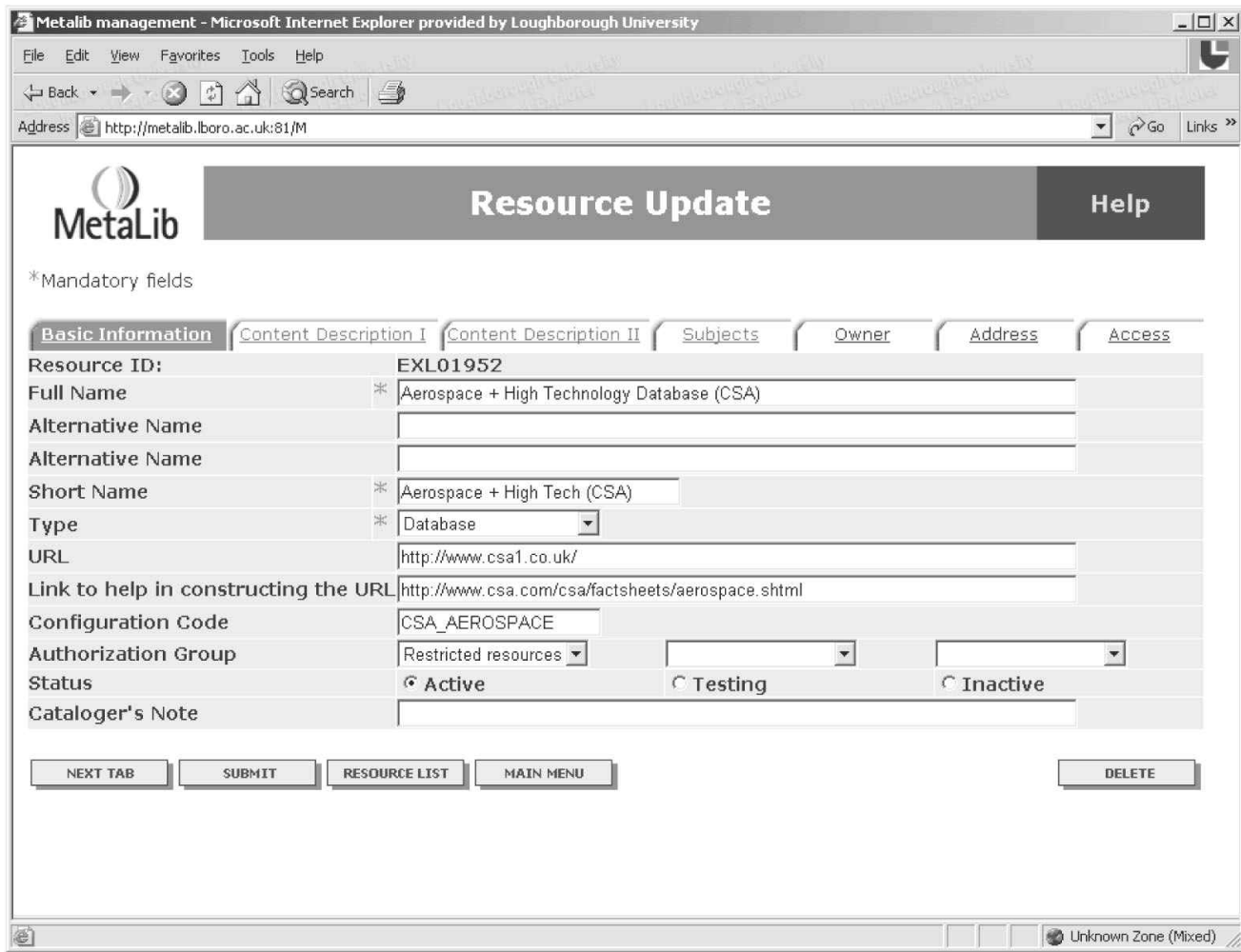


and, for those that have not been configured, they provide standard configuration forms (see Figure 3). Configuration has been problematical at Loughborough as no one team felt that it was their responsibility. The academic librarians felt it was either a support services or systems team task, while both those teams felt it was a role for the academic librarians. As it is part of the cataloguing process and requires basic knowledge of MARC, it was eventually agreed that it would be appropriate for the academic librarians to undertake this task. Unfortunately not many of the remaining databases are Z39.50-compatible; thus the academic librarians have not been able to practise the configuration of databases.

Ex Libris provided training in March. The training worked on the "live" service and, by the end of the three and a half days, 35 per cent of the databases were activated and 30 per cent configured for cross-searching (all OCLC databases to which the Library subscribed and most of CSA databases). During the next three months the academic librarians activated the remaining databases and catalogued from scratch approximately 50 per cent of the
Library's databases. This process could have been achieved more quickly with dedicated staff, but the academic librarians undertook the task in addition to their other duties.

Once all the resources were catalogued and configured on MetaLib, a month was spent testing the resources and quality-checking the catalogue records. Although the resources were tested, some of the searching quirks were only discovered when the service was live and being used by a large group of people. For example, INSPEC was returning the journal details in the imprint field not the source field and therefore the SFX service would not work correctly. Art Abstracts and EI Compendex do not always search as anticipated, as they do not allow phrase searching through Z39.50. Advice to other institutions implementing a similar service is "Test, test and test again!"

\section{Searching databases using MetaLib}

Readers log-on to MetaLib using their University user name and e-mail password. Once a reader has selected an information

Figure 3 Configuration record

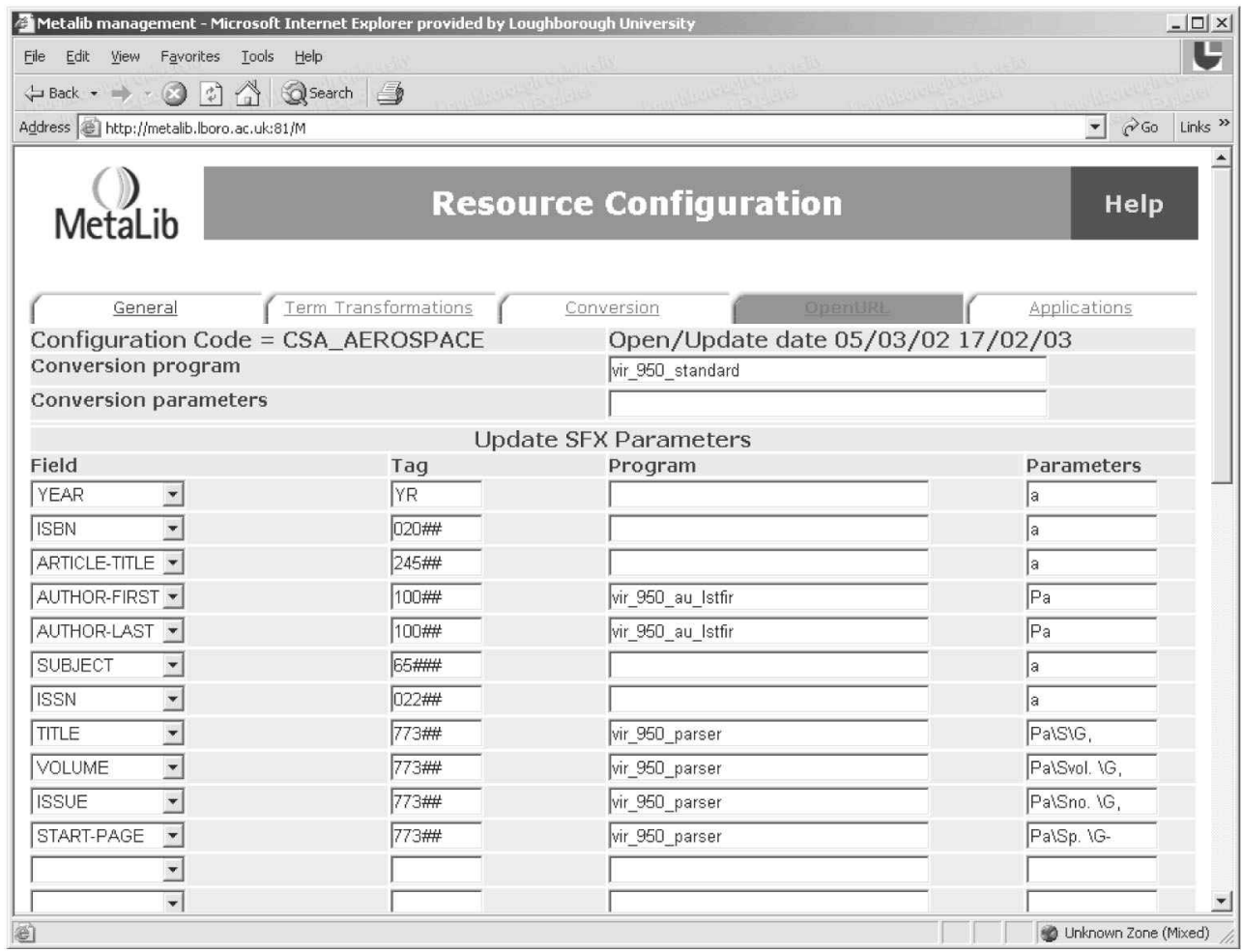


gateway, the search screen is divided into four. A list of information gateways is on the lefthand side of the screen. On the right-hand side is a search screen, a list of cross-searchable databases and a list of databases that can only be searched in their individual or "native" interfaces. Against the name of each database is an information icon that contains data about the coverage of the database, any authentication requirements and specific searching guidance.

If a reader wishes to search the "native" interface of a Web-based database, they just click on the hotlink and they are taken directly to the database in a separate window. If a reader wishes to search up to eight of the cross-searchable databases simultaneously, they need to check the boxes against the name of the databases and enter search term(s) in the search boxes provided.

Searches can be carried out using author, title or keyword. Boolean logic and truncation can also be used; however, some databases such as those provided by OCLC do not support truncation in the "native" interface and therefore do not support that function in Z39.50 searching.

The results list provides a brief record of each reference found, including the author, title of the article and date (see Figure 4). Feedback from the academic departments shows that this is one of the most negative features of MetaLib. They would prefer the journal title to be displayed at this point in time, as they use it as one of their evaluation criteria. Ex Libris do have plans to enhance the data displayed in the results list.

To view the full reference that normally includes an abstract, the reader clicks on the More button. It is at this stage that the SFX button is available, so that a reader can check whether the Library has a copy of the item (see Figure 5). MetaLib has similar functionality to any other database. A search can be refined, search results can be merged with duplicates being discarded and specific references can be added to a basket and then e-mailed or saved to disc.

\section{Readers and MetaLib}

In August 2002 the Library piloted the new service with three departments; the Business School, Civil Engineering, and Human Sciences. Focus groups were held with nine representatives from each department.

Participants were a mixture of academic staff, researchers and $\mathrm{PhD}$ students. Each focus group lasted for an hour and a half. During that time there was a demonstration of MetaLib, hands-on use of the service, completion of a questionnaire and group discussion. All participants liked the service, especially the cross-searching facility and feedback in general was positive:

- 99 per cent said MetaLib was easy to navigate round;

- 80 per cent said they had discovered new resources that they had not seen before, despite no new databases recently being purchased by the Library and the fact that 95 per cent of them used databases at least once a month or more;

- 100 per cent liked the look and feel of MetaLib;

- 50 per cent had never used the subject guides provided in print and electronic format by the Library; and

- 70 per cent thought MetaLib was an easier method of discovering what databases were available than the existing Library database pages.

Negative feedback related to the fact that not all databases are cross-searchable and, as discussed earlier, that journal titles do not appear in the brief results list (see Figure 4).

By the beginning of August all planned resources were catalogued on MetaLib and 35 per cent of them were cross-searchable. The service went live on the Library's Web pages and was actively advertised in September. Three training sessions were offered to all students and staff and over 60 people attended them.

Academic librarians also offered training directly to the academic departments and at least five out of 20 departments arranged for training to take place down in their departments.

Feedback has been very positive. Comments include "a very useful resource", "I did not know so many databases were available for my subject" and "I love playing with MetaLib".

\section{Statistics and usage of MetaLib}

Unfortunately MetaLib does not provide statistics on usage of MetaLib or databases 
Figure 4 Results list

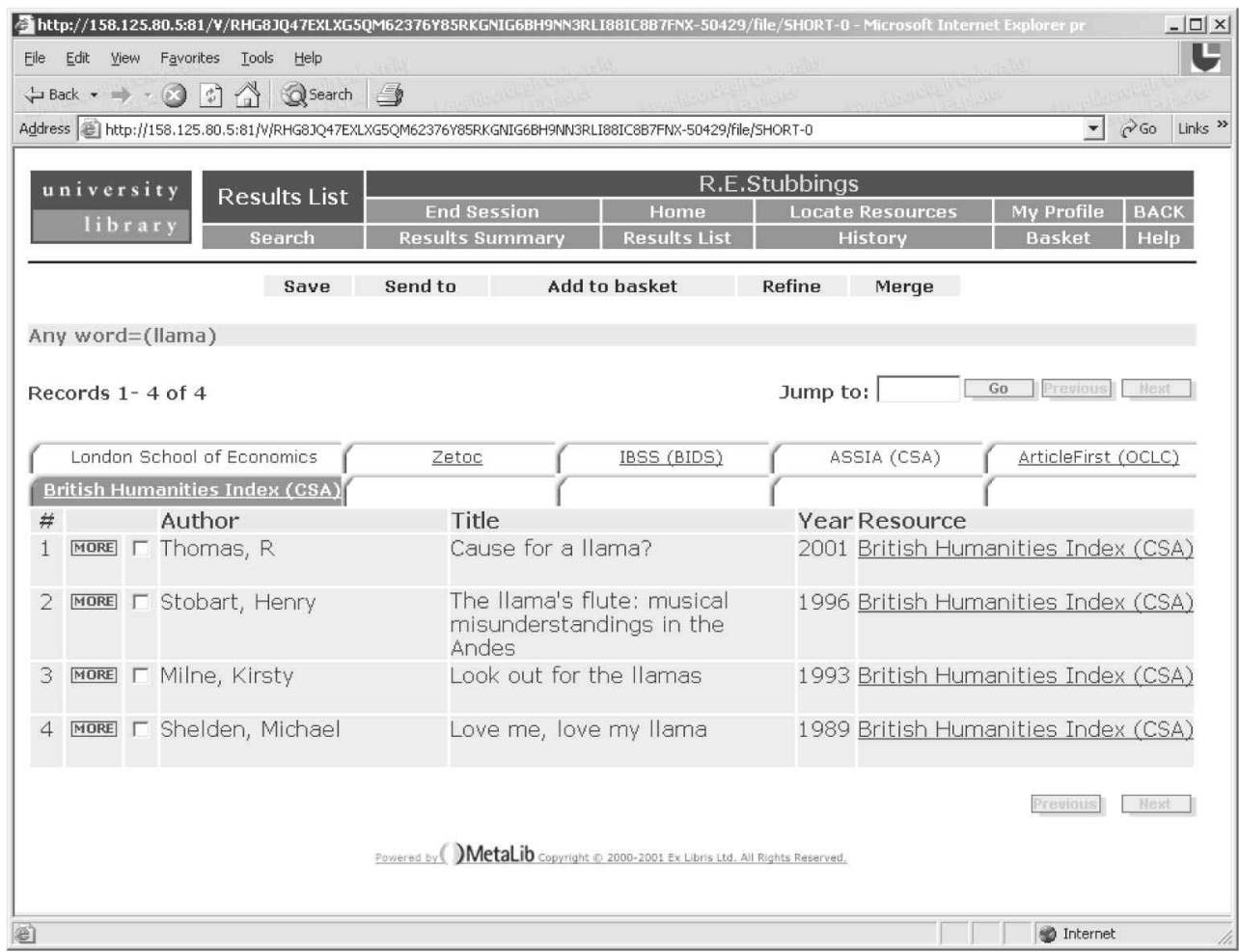

Figure 5 Full bibliographic record

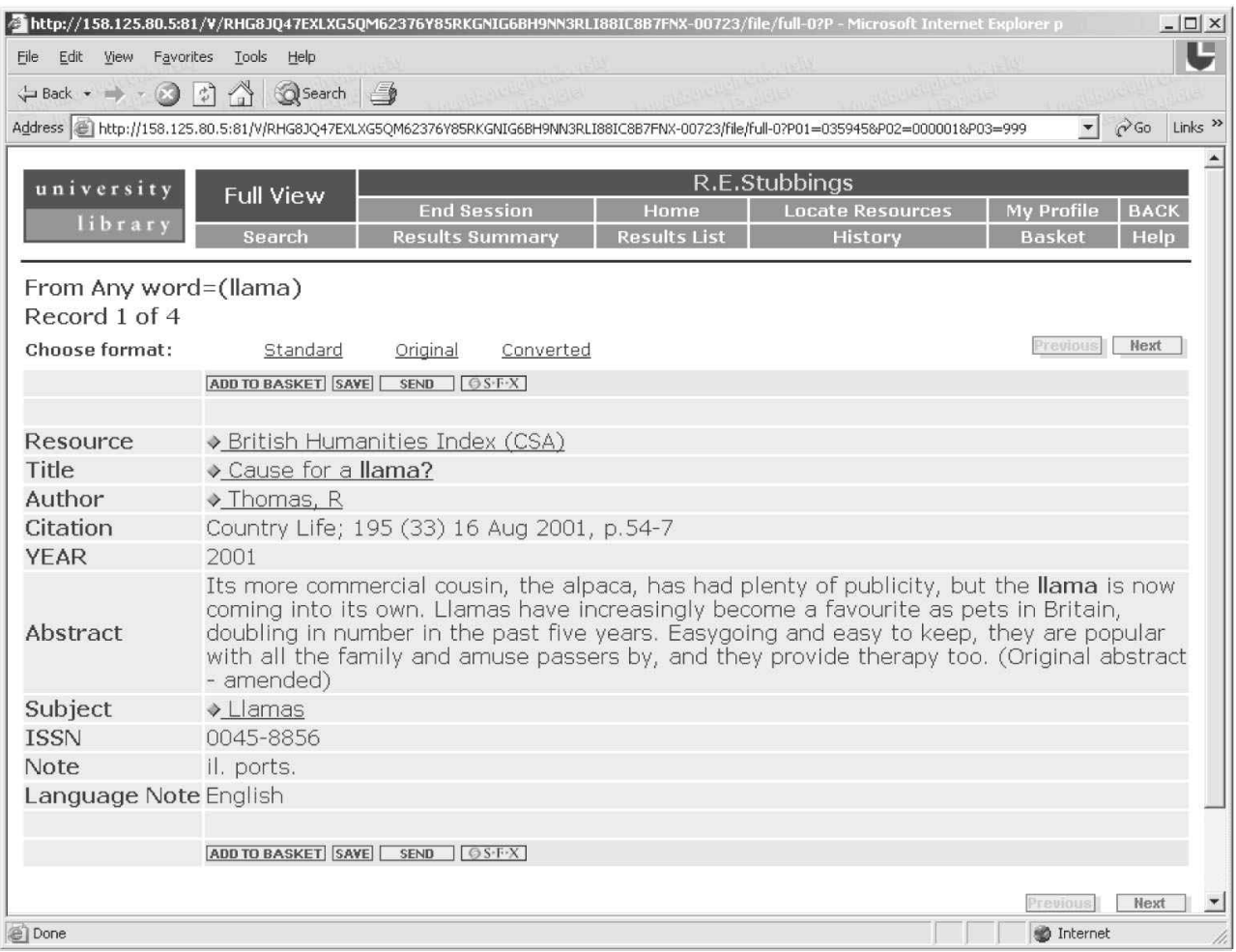


accessed via MetaLib. Ex Libris recognises this is not satisfactory and has plans to improve the situation. Luckily many suppliers provide statistics on direct and Z39.50 searches of their databases. As Loughborough University Library has always collated statistics on the usage of databases, there was an existing mechanism that could be utilised to collate data on Z39.50 searching. Comparisons of the available statistics is not always robust, as some suppliers provide data on the number of times a database is accessed, while others provide data on the number of searches carried out. Despite this, the statistics have been surprising. With the introduction of MetaLib, the Library has seen a growth in usage of the databases. Some have risen by 10 per cent, while others have risen more dramatically by 300 per cent. Although the cross-searchable databases have witnessed the greatest increases in usage, the remaining databases have also been used more as they have become more visible to the readers.

\section{Implementation of SFX}

The SFX Group was a small team of five, including representatives from the Systems, Support Service and Faculty Teams. Training was received in April and work began immediately on bringing the service online. SFX was relatively easy to set up, but it was a time-consuming process as both the journals and databases had to be made "SFXcompliant".

SFX has a knowledge base of targets (items to which the Library wishes to link, e.g. Library holdings of print journals or full text e-journals) (see Figure 6). In some instances it was just a case of activating the journal or supplier on the knowledge base. In others, it was more complicated, as details of the e-journals (title, publisher, ISSN, holdings and authentication procedures) had to be uploaded on to the knowledge base using Excel spreadsheets. Although all this information was available, it was not stored together in one place. Therefore it was a slow and time-consuming process transferring the data to the spreadsheets. Uploading the data once they were collated was a very quick and easy procedure. Ex Libris provide monthly updates to the knowledge base.
VINE: The Journal of Information and Knowledge Management Systems Volume $33 \cdot$ Number $1 \cdot 2003 \cdot 25-32$

Figure 6 SFX screen

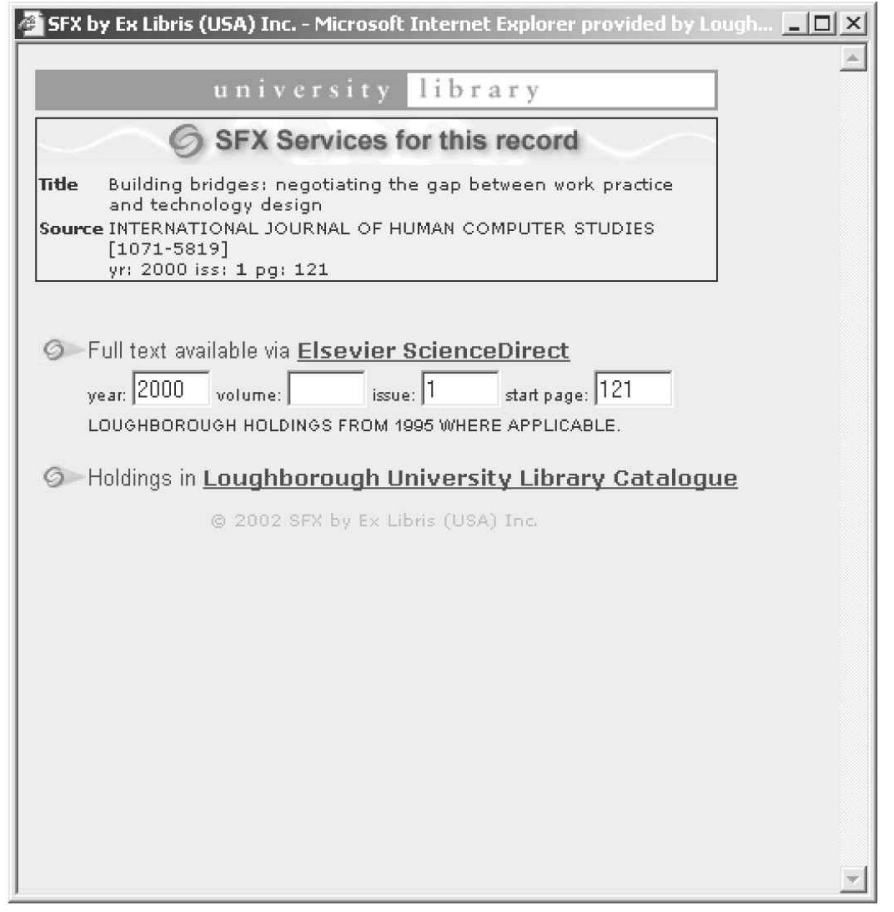

The setting-up of the databases to utilise SFX was more complex than first anticipated. Ex Libris provided documentation on how to set up major suppliers, e.g. CSA and Web of Science, but they did not always work in the prescribed way. Where there was no documentation or problems were experienced, the suppliers were helpful in providing assistance on how to link via SFX from their databases to external resources. The Library would like SFX to be activated on all the databases that are purchased. Therefore letters were sent to all suppliers asking if they were SFX-compliant. It was surprising how few knew about the technology and required additional data. Quite a few said they were not OpenURL-compliant but it would be something into which they would look.

SFX went live in July 2002 and to date approximately nearly all of the Library's journals are uploaded and activated on the knowledge base and are therefore accessible via SFX. Of the Library's database suppliers, only five have been SFX-enabled - all databases available from Cambridge Scientific Abstracts, OCLC, Web of Science, Emerald Abstracts, SWETS and MetaLib. This makes up approximately 35 per cent of the databases to 
which the Library subscribes. Unfortunately SFX does not work well when trying to link to books or conference papers and will often indicate that items are not in stock in the Library when they actually are. This is connected to the type of data that are being sent by the databases to the SFX server. Library staff were initially anxious that readers would be frustrated when the SFX button did not always link to the full text of an e-journal or the Library catalogue for the print subscription. However, reactions from the readers have been positive, with most being pleased to be able to discover whether a reference is available from the Library or not from within a database.

\section{The future}

The MetaLib Group is now only made up of four members of staff, a representative from the Systems Team, Support Services Team and two academic librarians. The main role of the group is to catalogue and configure new databases as they are purchased and to sort out problems when a database does not work as anticipated when undertaking a Z39.50 search. Currently the team spends approximately 5 per cent of a week working on MetaLib.

The Library has just been upgraded to version 2 of MetaLib, although the service is currently not available to the readers. Version 2 addresses issues relating to the number of information gateways in which a database can appear, use of icons and how databases are presented in each gateway. It also has a new "look and feel". Library staff like the new interface and are currently discussing possible local changes before the service goes live later this year.

The Support Services Team is working steadily to upload all the Library's e-journal subscriptions to the SFX server and this should be complete by late summer 2003 .

\section{Notes}

1 www.aleph.co.il/

2 www.aleph.co.il/metalib/index.html

3 www.sfxit.com/

4 http://lisweb.curtin.edu.au/electronicresources/ index.html

\section{References}

Cox, A. and Yeates, R. (2002), Library-Oriented Portals Solutions, Technology and Standards Watch Reports 02-03, JISC, London, available at: www.jisc.ac.uk/ uploaded_documents/tsw_02-03.pdf (accessed 17 February).

Lewis, N. (2002), "Talking about a revolution? First impressions of Ex Libris's MetaLib", Ariadne, No. 32, available at: www.ariadne.ac.uk/issue32/metalib/ (accessed 17 February). 